\section{PSICOLOGIA IBEROAMERICANA}

\section{Psicología lberoamericana}

ISSN: 1405-0943

psicología.iberoamericana@uia.mx

Universidad Iberoamericana, Ciudad de

México

México

Robles Ojeda, Francisco Javier; Sánchez Velasco, Alejandra; Galicia Moyeda, Iris Xóchitl Relación del Bienestar Psicológico, Depresión y Rendimiento Académico en Estudiantes de Secundaria

Psicología Iberoamericana, vol. 19, núm. 2, julio-diciembre, 2011, pp. 27-37

Universidad Iberoamericana, Ciudad de México

Distrito Federal, México

Disponible en: http://www.redalyc.org/articulo.oa?id=133921440004

Cómo citar el artículo

- Número completo

- Más información del artículo

- Página de la revista en redalyc.org

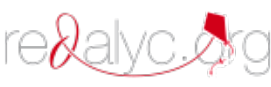

Sistema de Información Científica

Red de Revistas Científicas de América Latina, el Caribe, España y Portugal

Proyecto académico sin fines de lucro, desarrollado bajo la iniciativa de acceso abierto 


\title{
Relación del Bienestar Psicológico, Depresión y Rendimiento Académico en Estudiantes de Secundaria
}

\author{
Relationship of Psychological Well-being, Depression and Academic \\ Achievement in High-school Students
}

\author{
Francisco Javier Robles Ojeda \\ Alejandra Sánchez Velasco \\ Iris Xóchitl Galicia Moyeda \\ Facultad de Estudios Superiores Iztacala, México*
}

\section{RESUMEN}

La adolescencia presenta uno de los mayores niveles de prevalencia de depresión, afectando diversos ámbitos de la vida de quienes pasan por esta etapa, entre los que destaca el escolar. Para una mayor comprensión de la depresión, se sugiere indagar no sólo los factores de riesgo, sino también los llamados salutogénicos, como las emociones positivas, que han sido señalados como factores protectores de estados emocionales negativos. El objetivo de esta investigación es conocer la relación entre bienestar psicológico, depresión y rendimiento académico en estudiantes de educación secundaria e identificar diferencias entre hombres y mujeres. La muestra se conformó por 114 adolescentes de los tres grados de educación secundaria. Se aplicó el Inventario de Depresión de Kovacs, la adaptación al castellano de la Escala de Bienestar Psicológico de Riff, y el rendimiento escolar se evaluó mediante el promedio final de calificación de los estudiantes. Los resultados muestran diferencias entre hombres y mujeres en el rendimiento escolar y en los niveles de correlación de este parámetro con la depresión y el bienestar psicológico.

Descriptores: Depresión, bienestar psicológico, rendimiento escolar, adolescencia, salud.

\section{ABSTRACT}

Adolescence presents one of the highest levels of prevalence of depression, affecting several areas of his life, most notably, the school. For a better understanding of depression is suggested to investigate the role that positive or salutogenic factors. The objective of this research is to understand the relationship between psychological wellbeing, depression and academic achievement in secondary education and identify differences between men and women. The sample consisted of 114 adolescents from all three grades of secondary education. Was applied Depression Inventory Kovacs, Castilian adaptation of the Psychological Well-being Scale Riff and school performance was evaluated using the final average score of the students. The results show differences between men and women in school performance and levels of correlation between this parameter with depression and psychological well-being.

Keywords: Depression, psychological wellbeing, educational achievement, adolescence, health

\footnotetext{
* Para correspondencia: Francisco Javier Robles Ojeda. Correo electrónico: solucion20@hotmail.com Tel. 57810811/5522124867; Alejandra Sánchez Velasco. Correo electrónico: aavale@servidor.unam.mx Tel. 53915272; Iris Xóchitl Galicia Moyeda. Correo electrónico: iris@servidor.unam.mx Tel.: 55610758. Académicos de la Facultad de Estudios Superiores Iztacala, UNAM.
} 


\section{INTRODUCCIÓN}

De acuerdo al Instituto Nacional de Salud Mental de Estados Unidos, a escala mundial los trastornos de esta área de la salud representan cuatro de las 10 causas principales de discapacidad en las economías de mercado establecidas (Rush Universitary Medical Center, 2011). Este nivel de presencia ha llevado a que instituciones como la Organización Mundial de la Salud (OMS) planteen como una prioridad la investigación, prevención y tratamiento de las enfermedades mentales (OMS, 2004). Entre las que destacan se encuentra la depresión, que, de acuerdo con la OMS, se ubica como la cuarta causa mundial de morbilidad y se estima que será la segunda causa para el año 2020 (Medina-Mora et al., 2003; OMS, 2011; Pardo, Sandoval \& Umbarita, 2004).

Una de las etapas de mayor riesgo para el desarrollo de la depresión es la adolescencia, la cual presenta un nivel de prevalencia mayor con respecto a otras. $\mathrm{Al}$ igual que demás trastornos mentales, la depresión afecta de manera negativa el rendimiento óptimo que pudieran tener las personas en sus actividades cotidianas, por lo que en la adolescencia es importante analizar cómo influye en el desempeño en la escuela, ya que es un ámbito donde los adolescentes transcurren el mayor tiempo de su vida cotidiana. Al respecto, varios estudios han reportado una relación entre síntomas depresivos y problemas escolares como bajo rendimiento escolar, reprobación y deserción escolar (Campo-Arias, González, Sánchez, Rodríguez, Dallos \& Díaz-Martínez, 2005; Díaz, Prados \& López, 2002; Galicia, Sánchez \& Robles, 2007; Pérez \& Urquijo, 2001).

Ser hombre o mujer es otra variable a considerar en el estudio de los trastornos depresivos y sus efectos. De acuerdo con el DSM-IV (American Psychiatric Association, 1995), el trastorno depresivo mayor, tanto en adolescentes como en adultos, es dos veces más frecuente en mujeres que en varones, aunque en la edad prepuberal los niños y las niñas están afectados en la misma proporción. Lang y Tisher (1997) también encontraron porcentajes similares en la ado-

${ }^{1}$ Estimaciones de la OMS basadas en los niveles de prevalencia e incidencia del promedio de los años que una persona vive con una determinada enfermedad, y por el número de años potenciales de vida perdidos por muerte prematura debido a dicha enfermedad. lescencia temprana, aunque esta similitud se extendía sólo hasta los 16 años, y posteriormente se reportaba una disminución en los varones. Por su parte, Cabaco (1995) concuerda en la presencia de menores índices de depresión en varones, en específico en los que residen en el área urbana. En México, algunas encuestas realizadas con población adulta han encontrado una prevalencia del trastorno depresivo de entre 3 y $4.5 \%$, y reportan una mayor proporción -2 a $1-$ en la población femenina con respecto a la masculina (Bello, Puentes-Rosas, Medina-Mora \& Lozano, 2005). Leyva, Hernández, Nava y López (2007) mencionan otros estudios donde se contabilizan a sujetos con síntomas depresivos; se encontró una presencia de éstos en un rango de $22 \%$ a $50 \%$ de la población estudiada, y en su propia investigación - realizada en el estado de Guanajuato con adolescentes entre 15 y 19 años-, identificaron rasgos depresivos en $18 \%$ de los varones y $42 \%$ de las mujeres de la población investigada. De acuerdo con esta asociación encontrada, se podría esperar que el grupo de mujeres presentaran mayores problemáticas en el contexto escolar que los hombres, por ser en ellas en quienes prevalecen más los trastornos asociados a la depresión, sin embargo, los datos muestran que son precisamente las mujeres quienes tienen un mejor rendimiento escolar que los hombres en sus primeros años de trayectoria escolar (Cervini \& Dari, 2009; Gomez, 2000). En cuanto a la diferencia entre hombres y mujeres que presenta la asociación entre depresión y rendimiento escolar, no existen resultados claros al respecto, ya que varios estudios reportan resultados contradictorios con respecto a qué grupo presenta mayor correlación, o si no hay diferencias entre ellos. Para explicar esta falta de relación entre depresión y rendimiento académico, algunos autores se refieren a que existe una mayor presión del medio social hacia las mujeres con respecto a su rendimiento escolar, además de una tendencia cognitiva a atribuir los fracasos a factores internos y a una "rumiación mental" caracterizada por una mayor permanencia de los pensamientos asociados a autoculparse por dichos fracasos -esto contrasta con los hombres, que atribuyen los fracasos más a factores externos, además de que no presentan una tendencia marcada a la "rumiación mental" (Cerezo \& Casanova, 2004; Seligman, 2008). Esto podría propiciar que, ante la más leve adversidad, 
se viera afectado el estado emocional de forma negativa -como es el caso de la depresión.

La investigación sobre salud mental ha estado caracterizada primordialmente por el estudio de las enfermedades mentales, como es el caso de la depresión. Sin embargo, en los últimos años, se ha señalado la necesidad de indagar factores positivos que funcionan como protectores para el desarrollo de trastornos del estado de ánimo (Hervás, Sánchez \& Vázquez, 2008; Labiano, 2007; OMS, 2004; Vázquez \& Hervas, 2008). De manera específica, la corriente de la Psicología Positiva ha desarrollado intervenciones con el propósito de incrementar diversas áreas positivas del ser humano -emociones positivas, sensación de esperanza y autoeficacia, relaciones positivas, etcétera-, las cuales han reportado niveles importantes de eficacia y eficiencia, ya sea de manera aislada o en combinación con otras intervenciones para el tratamiento de síntomas depresivos (Peterson \& Park, 2004; Seligman, Steen, Park \& Peterson, 2005; Sin \& Lyubomirsky, 2009). Dentro de esta línea de pensamiento se encuentran los estudios acerca del Bienestar Psicológico (BP), entre los que destaca el modelo de Carol Riff, que propone que el BP implica el desarrollo del potencial humano, conformado por seis dimensiones: auto-aceptación, relaciones positivas, autonomía, dominio del entorno, crecimiento personal, y propósito en la vida (Ryff, 1995; Vázquez, Hervas, Rahona \& Gómez, 2009). Algunas investigaciones han señalado relaciones entre el BP y el desempeño escolar en jóvenes y adolescentes; por ejemplo, en una investigación con estudiantes universitarios en España (Salanova, Martínez, Bresó, Llorens \& Grau, 2005) se encontró que el BP estaba relacionado significativamente con una sensación de autoeficacia académica, de satisfacción con los estudios, motivación positiva y con un bajo nivel de propensión al abandono de los estudios. En otro estudio realizado con estudiantes de nivel medio superior en México (Chávez, 2006), se encontró una correlación positiva entre rendimiento escolar y BP. Con respecto a las diferencias en el BP presentadas entre hombres y mujeres, no han sido significativas en gran parte de los estudios realizados (Chávez, 2006; Casullo, 2002).

La presente investigación tiene como objetivo indagar si existen diferencias entre hombres y mujeres entre el bienestar psicológico, el nivel de depresión y el rendimiento académico en una población de adolescentes que cursan la educación secundaria en el Estado de México, así como identificar la relación existente en estos factores.

\section{MÉTODO \\ Participantes}

El estudio se realizó a partir de la población de una escuela secundaria pública del Estado de México, donde se seleccionó una muestra aleatoria conformada por 114 adolescentes ( 59 mujeres y 55 hombres) de los tres grados de educación secundaria (46 alumnos de primer grado, 30 de segundo grado y 38 de tercer grado), cuya edad oscilaba entre los 12 y 16 años. Los estudiantes participaron de manera voluntaria, previa autorización del departamento de orientación escolar de la institución educativa.

\section{Instrumentos}

Inventario de Depresión de Kovacs (1992). Conformado por 27 reactivos, compuesto a su vez por tres enunciados (opciones A, B y C). Los alumnos tenían que seleccionar el que describiera mejor la forma en que se había sentido en las dos últimas semanas. Proporciona tres niveles de tendencia depresiva: leve ( 7 a 13 puntos), moderada (14 a 19 puntos) y severa ( $>19$ puntos). El nivel de confiabilidad reportado por la autora de este instrumento es de 0.86 .

Adaptación al castellano de la Escala de Bienestar Psicológico de Riff (Díaz, Rodríguez-Carvajal, Blanco, Moreno-Jiménez, Gallardo, Valle, \& Van Dierendonck, 2006). Consta de 39 enunciados basados en las seis dimensiones del BP del modelo de Carol Riff con seis opciones de respuestas distribuidas en una escala tipo Likert (de 1 "completamente de acuerdo," hasta 6 "completamente en desacuerdo"). Para el presente estudio, con base en los valores de la media y la desviación típica, se crearon cinco niveles de bienestar: muy bajo (0-2.2 puntos), bajo (entre 2.3-3.6 puntos) medio (3.7-4.2 puntos) alto (4.3-5 puntos) y muy alto ( $>5$ puntos). Los niveles de confiabilidad reportados para las escalas de este instrumento oscilan entre 0.71 y 0.83 .

Rendimiento escolar. Se evaluó mediante el promedio final (general y de cada una de las materias) de 
calificación correspondientes al ciclo escolar en curso (2008-2009).

\section{Procedimiento}

Previa autorización de los adolescentes participantes de la presente investigación, sus padres y los directivos de la institución educativa, se aplicó el Inventario de Depresión de Kovacs y la Escala de Bienestar Psicológico de Ryff. Para la administración de los instrumentos se dividió a la población en seis grupos de aproximadamente 20 sujetos cada uno, de acuerdo al grado escolar que cursaban. Se les proporcionó las instrucciones de cómo responderlos y se les dio un ejemplo; posteriormente, el aplicador iba leyendo uno a uno los reactivos de cada uno de los instrumentos mientras los alumnos seleccionaban la respuesta que mejor reflejaba su situación. El promedio general de los alumnos participantes se obtuvo a través de los archivos del departamento de Control Escolar de la escuela a la que pertenecían.

\section{RESULTADOS}

Los datos recabados fueron analizados con el programa estadístico SPSS, versión 17. Para conocer la existencia de diferencias entre mujeres y hombres en las variables investigadas -depresión, bienestar psicológico y rendimiento escolar- se realizó un análisis descriptivo diferenciado por sexo, donde se presentó la distribución por edades de los puntajes promedio de los niveles de depresión, de bienestar psicológico y rendimiento escolar, y la distribución de acuerdo al promedio de calificación de los niveles de depresión y bienestar psicológico. Con el fin de identificar las diferencias estadísticamente significativas por sexo de las variables investigadas, se llevó a cabo además un análisis de diferencias de grupos a partir de la prueba " $t$ " de Student ${ }^{2}$. Para conocer la existencia de relación entre depresión, bienestar psicológico y rendimiento escolar se realizó un análisis de correlación bivariada a través de la prueba " $r$ " de Pearson.

${ }^{2}$ En el apartado donde se explican los resultados de los "Análisis de diferencia de grupo por sexo" se mencionará los resultados de la prueba " $t$ " de Student, sólo en los casos que se encontraron diferencias estadísticamente significativas.
Análisis de diferencia de grupos de acuerdo al sexo Los resultados indican que la mayor parte de los adolescentes evaluados presentan rasgos depresivos, siendo los niveles leves y severos los que tienen mayores porcentajes de presencia. Con el fin de identificar las diferencias entre hombres y mujeres en los niveles de depresión se muestra la distribución de dichos niveles entre ambos sexos, donde se observan porcentajes semejantes en los niveles normal y moderado de depresión, mientras que en el nivel leve hay un porcentaje mayor en los hombres, y en el severo son las mujeres quienes lo tienen (ver Tabla 1).

Tabla 1. Distribución de los niveles de depresión en la muestra total, en mujeres y hombres

\begin{tabular}{cccc}
\hline $\begin{array}{c}\text { Nivel de } \\
\text { depresión }\end{array}$ & $\begin{array}{c}\text { Porcentaje } \\
\text { (muestra total) }\end{array}$ & $\begin{array}{c}\text { Porcentaje } \\
\text { (mujeres) }\end{array}$ & $\begin{array}{c}\text { Porcentaje } \\
\text { (hombres) }\end{array}$ \\
\hline Normal & 22.3 & 24.1 & 20.8 \\
Leve & 39.3 & 32.8 & 45.3 \\
Moderada & 16.1 & 15.5 & 17.0 \\
Severa & 22.3 & 27.6 & 17.0 \\
\hline
\end{tabular}

En cuanto a la distribución por edades, los promedios más altos de depresión se presentan en las edades más tempranas de la muestra, los cuales tienden a decrecer conforme el individuo va ascendiendo en edad, a excepción de los 16 años en la población de mujeres, donde se eleva de manera considerable (ver Gráfica 1).

\section{Gráfica 1. Nivel de depresión acuerdo al sexo y edad}

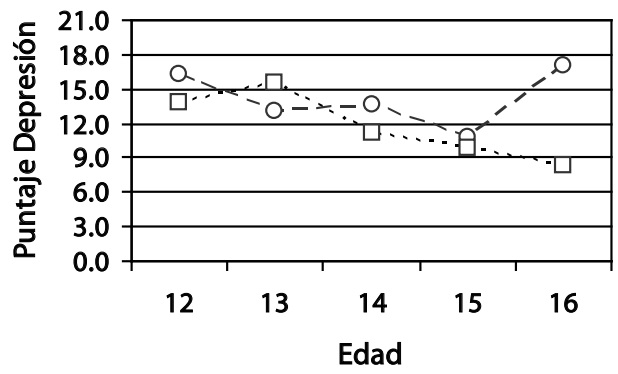

$--\mathrm{O}-$ - Mujeres ...- - - Hombres 
En relación al $\mathrm{BP}$, la mayor parte de la población se concentra en los niveles alto y medio. En cuanto a la diferencia de acuerdo al sexo, no se encontraron diferencias estadísticamente significativas en el promedio total de BP ni en alguna de sus subescalas. Con respecto a la distribución de los niveles de BP, hay una mayor presencia en las mujeres de los niveles alto, muy alto y muy bajo, mientras que en los hombres se observa una mayor presencia de niveles medio y bajo (ver Tabla 2).
En lo que toca al rendimiento escolar, la media del promedio final de calificaciones es 7.7. Las mujeres tienen una media de 8.1 y los hombres de 7.2, lo que representa una diferencia estadísticamente significativa entre ambos grupos $(t=4.779, p=.000)$. El promedio de calificaciones presenta una tendencia a la baja conforme aumenta la edad de los estudiantes, a excepción de los 16 años en las mujeres y de los 15 en los hombres, donde aumenta levemente con respecto a la edad

Tabla 2. Porcentaje de la población total, mujeres y hombres en los niveles de Bienestar Psicológico

\begin{tabular}{|l|c|c|c|}
\hline Nivel de Bienestar Psicológico & Porcentaje (muestra total) & Porcentaje (mujeres) & Porcentaje (hombres) \\
\hline Muy alto & 17.5 & 20.3 & 14.8 \\
\hline Alto & 37.7 & 39.0 & 37.0 \\
\hline Medio & 28.9 & 25.4 & 33.3 \\
\hline Bajo & 14.0 & 11.9 & 14.8 \\
\hline Muy bajo & 1.8 & 3.4 & 0.00 \\
\hline
\end{tabular}

Con respecto a la distribución del $\mathrm{BP}$ por edades, el promedio se concentra en el rango de 3.2 a 3.7, manteniéndose constante en todos los grupos, sin existir en ninguno de ellos una diferencia significativa entre hombres y mujeres (ver Gráfica 2).

Gráfica 2. Nivel de bienestar por sexo y edad

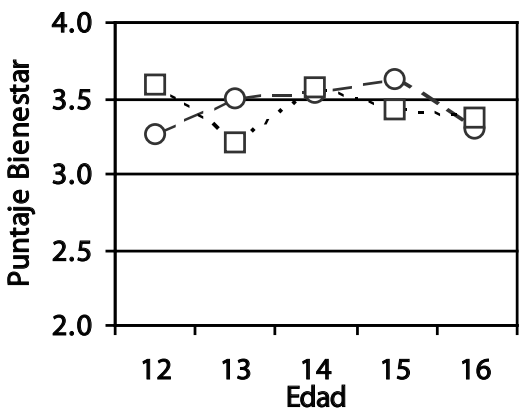

$$
\begin{aligned}
& \text { - - - - Mujeres } \\
& \text { - - - - - Hombres }
\end{aligned}
$$

anterior. En todas las edades las mujeres muestran un mejor rendimiento escolar que los hombres aunque, conforme van creciendo, esta diferencia se va reduciendo e incluso ya no es significativa estadísticamente en los grupos de 15 y 16 años (ver Gráfica 3).

Gráfica 3. Promedio de calificaciones por sexo y edad

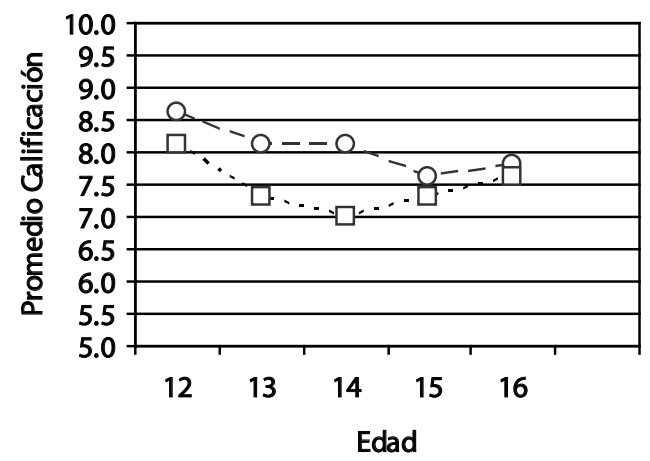


En cuanto a la relación entre los niveles de depresión y el rendimiento escolar, podemos apreciar una tendencia a que los niveles de depresión disminuyan en tanto el promedio de calificación aumenta -a excepción del promedio de 10-; por ejemplo, los datos obtenidos permiten apreciar que los alumnos con promedio general 6 presentan un nivel de depresión moderada (14-19 puntos), mientras que los que tienen promedio de 9 o 10 tienen puntajes medios o mínimos del nivel de depresión leve (7-13 puntos) (ver Gráfica 4).

Gráfica 4. Nivel de depresión de acuerdo al sexo y calificación

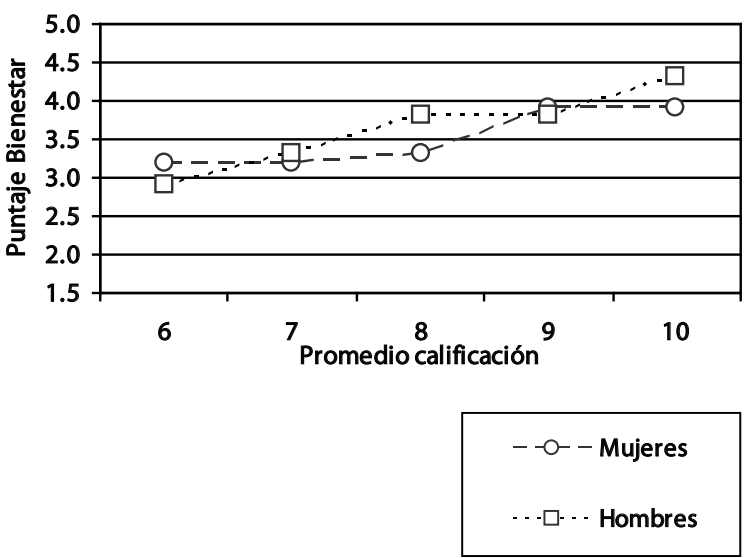

En cuanto al Bienestar Psicológico, se puede observar un aumento conforme aumenta el promedio de calificación. De manera general, se observa que los alumnos con un promedio de 6 se encuentran en el nivel de bienestar medio (3.7-4.2), mientras que los alumnos con 9 o 10 se encuentran en un nivel de bienestar alto (4.3-5) (ver Gráfica 5).

\section{Análisis de correlación}

Un análisis de correlación muestra una asociación estadísticamente significativa entre las tres variables investigadas en este estudio en la población total (ver Tabla 3). Entre los puntajes de depresión y BP se encontró una correlación negativa y moderada $(r=-.576$, $p=.001)$ y una correlación negativa de nivel bajo entre la depresión y el promedio de las calificaciones $(r=-.218, p=.021)$. La relación entre el rendimiento escolar y el bienestar psicológico es moderada y positiva $(r=.404, p=.001)$

En la población femenina se encuentra una correlación alta y negativa $(r=-.719, p=.001)$ entre depresión y bienestar, moderada y positiva $(r=.404, p=.002)$ entre bienestar y rendimiento escolar, sin embargo no hay una correlación significativa $(r=-.258, p=.055)$ entre depresión y rendimiento escolar. En la población masculina se encuentra una correlación moderada y negativa ( $r=-.361, p=.008)$ entre depresión y bienestar, así como entre depresión y rendimiento escolar ( $r=-.367, p=.007)$, mientras que entre bienestar y rendimiento escolar se presentó una correlación moderada y positiva $(r=.482, p=.000)$ (ver Tabla 3 ).

Correlación entre las subescalas de la Escala de Bienestar Psicológico y los factores del Inventario de Kovacs. Se encontraron correlaciones significativas entre todas las subescalas del BP y los factores del Inventario de Kovacs en la población total y en la de mujeres. En los hombres no existió una relación significativa entre las subescalas de Autonomía y Relaciones Personales con los factores del Inventario de Kovacs y entre el Dominio del Entorno y los factores de Falta de Entusiasmo y Depresión (ver Tabla 4).

Gráfica 5. Nivel de bienestar de acuerdo al sexo y calificación
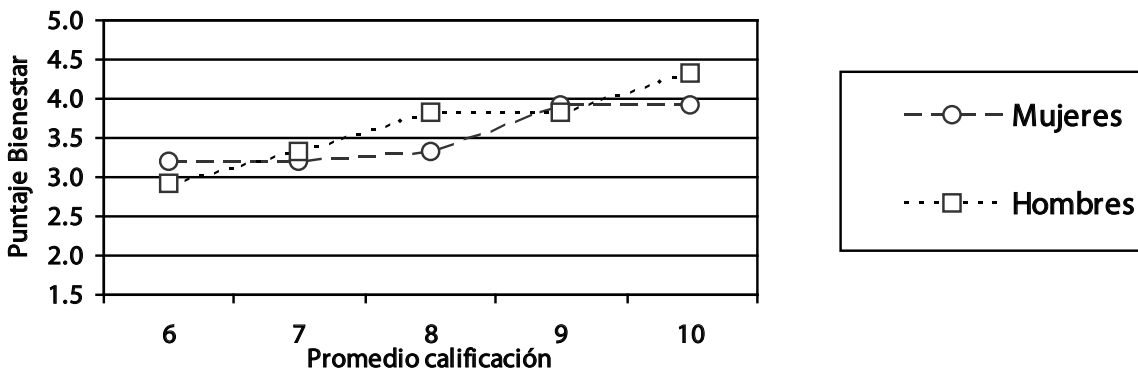
Tabla 3. Correlación entre Depresión, BP y Rendimiento Escolar en la población total, mujeres y hombres

\begin{tabular}{lccc}
\hline Par de variables correlacionadas & Población total & Mujeres & Hombres \\
\hline Depresión-Bienestar Psicológico & $-.576^{* *}$ & $-.719 * *$ & $-.361 * *$ \\
Depresión-Rendimiento escolar & $-.218^{*}$ & -.258 & $-.367 * *$ \\
Bienestar Psicológico-Rendimiento escolar & $.404 * *$ & $.404 * *$ & $.482 * *$ \\
\hline
\end{tabular}

Tabla 4. Correlación entre las subescalas de la Escala de Bienestar Psicológico y los factores del Inventario de Kovacs en la población total, mujeres y hombres

\begin{tabular}{|c|c|c|c|c|c|c|c|c|c|c|c|c|}
\hline FIK & \multicolumn{3}{|c|}{ Total Kovacs } & \multicolumn{3}{|c|}{ Falta de entusiasmo } & \multicolumn{3}{|c|}{ Depresión } & \multicolumn{3}{|c|}{ Relaciones interpersonales } \\
\hline SBP & PbTotal & $\mathrm{PbM}$ & $\mathrm{PbH}$ & PbTotal & $\mathrm{PbM}$ & $\mathrm{PbH}$ & PbTotal & $\mathrm{PbM}$ & $\mathrm{PbH}$ & PbTotal & $\mathrm{PbM}$ & $\mathrm{PbH}$ \\
\hline TBP & $-.576^{* *}$ & $-.719 * *$ & $-.361^{* *}$ & $-.499 * *$ & $-.602^{* *}$ & $-.320^{*}$ & $-.519 * *$ & $-.668^{* *}$ & $-.325^{*}$ & $-.524^{* *}$ & $-.688^{* *}$ & $-.298^{*}$ \\
\hline $\mathrm{RP}$ & $-.522^{* *}$ & $-.625^{* *}$ & $-.484^{* *}$ & $-.471^{* *}$ & $-.540^{* *}$ & $-.452^{* *}$ & $-.468^{* *}$ & $-.571 * *$ & $-.476^{* *}$ & $-.463^{* *}$ & $-.594 * *$ & $-.337^{*}$ \\
\hline AUT & $-.278^{* *}$ & $-.449 * *$ & -.028 & $-.209^{*}$ & $-.338^{* *}$ & .012 & $-.297^{* *}$ & $-.446^{* *}$ & -.104 & $-.218^{*}$ & $-.422^{* *}$ & .037 \\
\hline $\mathrm{DE}$ & $-.474^{* *}$ & $-.614^{* *}$ & $-.320^{*}$ & $-.402^{* *}$ & $-.511^{* *}$ & -.262 & $-.427^{* *}$ & $-.602^{* *}$ & -.242 & $-.439 * *$ & $-.544^{* *}$ & $-.335^{*}$ \\
\hline PV & $-.440^{* *}$ & $-.576^{* *}$ & $-.275^{*}$ & $-.396^{* *}$ & $-.514^{* *}$ & -.227 & $-.350^{* *}$ & $-.483 * *$ & -.204 & $-.452^{* *}$ & $-.596^{* *}$ & $-.290^{*}$ \\
\hline $\mathrm{CP}$ & $-.313^{* *}$ & $-.481^{* *}$ & -.048 & $-.288^{* *}$ & $-.417^{* *}$ & -.068 & $-.273^{* *}$ & $-.458 * *$ & -.017 & $-.282^{* *}$ & $-.429 * *$ & -.088 \\
\hline$A C$ & $-.564^{* *}$ & $-.702 * *$ & $-.322^{*}$ & $-.477 * *$ & $-.559 * *$ & $-.322 *$ & $-.525 * *$ & $-.640 * *$ & $-.334 *$ & $-.500 * *$ & $-.716^{* *}$ & -.186 \\
\hline
\end{tabular}

FIK= Factores del Inventario de Kovacs; $\mathrm{SBP}=$ Subescalas de Bienestar Psicológico; PbTotal= Población Total; PbM= Población de Mujeres; $\mathrm{PbH}=$ Población de Hombres

$\mathrm{TBP}=$ Total de Bienestar Psicológico; $\mathrm{RP}=$ Relaciones Positivas; $\mathrm{AUT}=$ Autonomía; $\mathrm{DE}=$ Dominio del Entorno; $\mathrm{PV}=\mathrm{Propósito} \mathrm{en} \mathrm{la} \mathrm{Vida;} \mathrm{CP}=$ Crecimiento Personal; AC= Aceptación

Correlación entre los factores del Inventario de Kovacs y el Rendimiento Escolar. Tanto en la población total como en la de hombres se encontró una relación significativa entre los factores de Falta de Entusiasmo y Relaciones Interpersonales con el Rendimiento Escolar, mientras que en las mujeres no hubo una relación significativa en ninguno de los tres factores del Kovacs (ver Tabla 5).

Tabla 5. Relación del rendimiento escolar con los factores del Inventario de Kovacs en la población total, mujeres y hombres

\begin{tabular}{lccc}
\hline \multicolumn{3}{c}{ Rendimiento escolar (promedio general de calificación) } \\
\hline Factores Kovacs & Población total & Mujeres & Hombres \\
\hline Total Kovacs & $-.218\left(^{*}\right)$ & -.258 & $-.367\left(^{* *}\right)$ \\
Falta de entusiasmo & $-.217\left(^{*}\right)$ & -.229 & $-.374\left(^{* *}\right)$ \\
Depresión & -.119 & -.230 & -.175 \\
Relaciones interpersonales & $-.281\left(^{* *}\right)$ & -.250 & $-.443\left(^{* *}\right)$ \\
\hline
\end{tabular}


Correlación entre las subescalas de la Escala de Bienestar Psicológico y el Rendimiento Escolar. El análisis estadístico muestra una relación significativa en la población total de todas las subescalas de BP con el rendimiento escolar. En las mujeres sólo la dimensión de Relaciones no presentó una correlación significativa, mientras que en el caso de los hombres no se presentó en las dimensiones de Aceptación, Autonomía y Dominio del Entorno (ver Tabla 6).

\section{DISCUSIÓN}

Los mayores niveles de depresión encontrados en el grupo de mujeres del presente estudio coinciden con investigaciones realizadas en nuestro país, donde se señala un mayor índice de depresión en el sexo femenino y en la adolescencia temprana (Medina-Mora et al., 2003; Pérez \& Urquijo, 2001). En ambos sexos se presentan mayores niveles de depresión en los grupos de edad más jóvenes -12 y 13 años-, los cuales decrementan conforme aumenta la edad, a excepción del grupo de mujeres de 16 años, donde aumenta, lo cual puede estar asociado a que la mayor parte de las alumnas en este grupo de edad tienen rezago escolar y un historial de reprobación. Autores como Díaz, Prados \& López (2002) señalan que los adolescentes que se mantienen en un nivel escolar a una edad en que se esperaría que ya estuvieran en un nivel superior se exponen a un mayor número de experiencias de fracaso y frustración, esto aunado a factores individuales que propician que las mujeres sean más susceptibles a los fracasos escolares, traería como consecuencia una mayor probabilidad de que sufran depresión.
Por su parte, el Bienestar Psicológico presentó una incidencia mayor en las mujeres -en específico, de los niveles "alto" y "muy alto", aunque no se encontraron diferencias significativas entre hombres y mujeres con respecto al promedio general del nivel de bienestar psicológico, lo cual coincide con otras investigaciones realizadas en Latinoamérica, donde tampoco hubo diferencias a nivel estadístico (Chávez, 2006; Casullo, 2002).

Con respecto al Rendimiento Escolar, se encontraron diferencias significativas entre hombres y mujeres, siendo ellas las que presentan un mejor índice de aprovechamiento escolar, tendencia que coincide con diversos estudios que mencionan que en las últimas décadas se ha encontrado de manera consistente un mejor rendimiento escolar de las mujeres, sobre todo en niveles de educación básica y media, lo que ha llevado incluso a que se hable de una "feminización" del éxito académico (Cervini \& Dari, 2009; Gómez, 2000).

A nivel estadístico, el factor de Bienestar Psicológico muestra índices similares de relación con el rendimiento escolar, tanto en hombres como en mujeres, y también en ambos grupos se presenta una relación significativa entre Bienestar Psicológico y depresión, presentándose en las mujeres niveles de relación más fuertes que en el grupo de hombres. En cuanto a la relación de los diversos factores del Bienestar Psicológico con la variable de depresión, se encontró en las mujeres una relación negativa y estadísticamente significativa entre todas las subescalas de los instrumentos que medían ambas variables, mientras que en el caso de los hombres sólo se presentó una relación entre el puntaje

Tabla 6. Relación del rendimiento escolar con las subescalas de la Escala de Bienestar Psicológico en la población total, mujeres y hombres

\begin{tabular}{lccc}
\hline \multicolumn{3}{c}{ Rendimiento escolar (promedio general de calificación) } \\
\hline Subescalas Bienestar & Población total & Mujeres & Hombres \\
\hline Total Bienestar Psicológico & $.404^{* *}$ & $.404^{* *}$ & $.482^{* *}$ \\
Relaciones positivas & $.308^{* *}$ & .170 & $.364^{* *}$ \\
Autonomía & $.275^{* *}$ & $.362^{* *}$ & .247 \\
Dominio del entorno & $.274^{* *}$ & $.341^{* *}$ & .263 \\
Propósito en la vida & $.353^{* *}$ & $.354^{* *}$ & $.453^{* *}$ \\
Crecimiento personal & $.349^{* *}$ & $.410^{* *}$ & $.391^{* *}$ \\
Aceptación & $.225^{*}$ & $.299^{*}$ & .214 \\
\hline
\end{tabular}


global de depresión y el de Bienestar Psicológico pero no entre sus subescalas. Estos resultados apoyarían la idea de que la tendencia ya mencionada de las mujeres a culparse a sí mismas por sus fracasos, y a mantener dichos pensamientos por más tiempo, propiciaría más fácilmente síntomas depresivos, los cuales, a su vez, impactarían más al bienestar psicológico.

Los resultados ya comentados sobre los factores de depresión y rendimiento escolar nos permiten apreciar diferencias importantes en la manera en que estas variables se relacionan en mujeres y hombres. A nivel estadístico, en los hombres se presenta una relación significativa de tipo negativo, es decir, entre mayor depresión exista hay menores índices de aprovechamiento escolar, mientras que en el grupo de mujeres no hubo ninguna relación estadísticamente significativa entre los componentes de estos dos factores -depresión y rendimiento escolar-. A nivel descriptivo, el grupo de mujeres es el que tiene mejores índices de aprovechamiento escolar; sin embargo, también son las que presentan mayores índices de depresión, mientras que el grupo de hombres tiene peores resultados en su aprovechamiento escolar, aunque sus índices de depresión son menores que el de las mujeres. Un señalamiento importante con relación a estos datos obtenidos es que un alto rendimiento escolar no puede ser considerado de manera general como un indicador de ausencia de depresión -como en el caso del grupo de mujeres del presente estudio.

Una posible explicación a estos resultados se encuentra en estudios que señalan una diferencia entre hombres y mujeres con respecto a la manera en que explican sus logros escolares. Dichas investigaciones muestran que los hombres no tienden a culparse a sí mismos de sus fracasos escolares -por ejemplo, el bajo rendimiento escolar- sino que suelen atribuirlos a factores externos -principalmente a los maestros y a la suerte (Cerezo \& Casanova, 2004)-. Aunado a esto, también se ha señalado que las conductas asociadas a un buen desempeño académico -por ejemplo, organización en los apuntes, dedicación al estudio o participación activa en clase- se han ido percibiendo como "femeninas", por lo que en algunos ámbitos y grupos de edades -sobre todo en grupos de adolescentes donde predominan comportamientos tradicionales de género- dichas conductas son poco valoradas para los va- rones adolescentes e, incluso, los adultos con los que conviven -familia y maestros- esperan que ellos se comporten de manera desorganizada, distraída, desmotivada y que sean rebeldes antes las figuras de autoridad y las normas y obligaciones escolares (Gómez, 2000). Esto propicia que el bajo rendimiento escolar en los varones no sea percibido como un evento "catastrófico" a nivel emocional y no genere los altos índices de depresión que presenta su contraparte femenina -a pesar de que ellas tienen mejor aprovechamiento escolar.

En el caso de las mujeres, hay una tendencia a atribuir los fracasos escolares a factores internos -por ejemplo, a tener poca habilidad o no haber realizado un suficiente esfuerzo-, lo que las lleva a culparse más fácilmente por bajas notas escolares (Cerezo \& Casanova, 2004). Esto explicaría por qué a pesar de que la presencia en el grupo de mujeres de mayores índices de aprovechamiento escolar, no se presenten, a su vez, menores índices de depresión que en los hombres, y que los niveles de bienestar sean semejantes en ambos grupos. Además, al contrario de los hombres, se espera de ellas un mejor comportamiento y un mayor rendimiento escolar (Gómez, 2000), por lo que en muchas ocasiones tienen que realizar un mayor esfuerzo para ser valoradas por los demás e incluso por ellas mismas. Esta diferencia entre los resultados obtenidos y la valoración de los demás hacia sus éxitos escolares podría explicar en parte la disminución en su aprovechamiento escolar conforme pasan los años de su educación secundaria, debido a que ellas mismas empieza a dejar de percibir al éxito escolar como algo valioso.

Estos resultados disímiles en los grupos de hombres y mujeres, así como su distinta relación con el rendimiento escolar, concuerdan con el señalamiento de varios autores (Díaz, Blanco, Horcajo \& Valle, 2007; Vázquez, 2009), quienes mencionan que, aunque es clara la relación entre el Bienestar Psicológico y la depresión, se tienen que entender como factores separados; por lo que se deberían generar no sólo estrategias para prevenir o minimizar los niveles de depresión, sino también para incrementar el primero, dando como resultado una atención integral a la salud mental del adolescente. Finalmente, se sugiere en futuros estudios abarcar grupos de escuelas y entornos donde el rendimiento académico y el nivel de exigencia por parte 
de los adultos sea similar, tanto para hombres como para mujeres, así como indagar el nivel de expectativas y autoeficacia con respecto al desempeño académico,

\section{REFERENCIAS}

Bello, M., Puentes-Rosas E., Medina-Mora M. E. \& Lozano, R. (2005). Prevalencia y diagnóstico de depresión en población adulta en México. Salud Pública, 5(47-1), 4-11.

Cabaco, A. S. (1995). Indicadores de riesgo y estrategias de prevención en la adolescencia. (Tesis Doctoral). Salamanca: Publicaciones de la UPSA.

Campo-Arias, A., González, S., Sánchez, Z., Rodríguez, D., Dallos, C. y Díaz-Martínez, L. (2005). Percepción de rendimiento académico y síntomas depresivos en estudiantes de media vocacional de Bucaramanga, Colombia. Archivos Pediátricos Uruguayos, 76(1), 21-26.

Casullo, M. (2002). Evaluación del Bienestar Psicológico en Iberoamérica. Buenos Aires: Paidós.

Cerezo, M. \& Casanova, P. (2004). Gender differences in academics motivation of secondary school students. Electronic Journal of Research in Education Psychology, 2(1), 97-112.

Cervini R. \& Dari, N. (2009). Género, escuela y logro escolar en matemática y lengua de la educación media. Revista Mexicana de Investigación Educativa, 14(42), 1051-1078.

Chávez, A. (2006). Bienestar psicológico y su influencia en el rendimiento académico de estudiantes de nivel medio superior. (Tesis de Maestría en Psicología Aplicada). Universidad de Colima.

Díaz, F., Prados, M. \& López, S. (2002). Relación entre rendimiento académico, síntomas depresivos, edad y género en una población de adolescentes. Psiquiatria. com, 6(2) 1-10.

Díaz, D., Blanco, A., Horcajo, J. \& Valle, C. (2007). La aplicación del modelo del estado completo de salud al estudio de la depresión. Psicothema, 19(2), 286-294.

Díaz, D., Rodríguez-Carvajal R., Blanco, A., MorenoJiménez, B., Gallardo, I., Valle, C. \& Van Dierendonck, D. (2006). Adaptación española de las escalas de bienestar psicológico de Ryff. Psicothema, 18(3), 572577. debido a que, como ya se señaló, son factores que pueden influir en la relación entre el rendimiento escolar con los niveles de depresión y Bienestar Psicológico.

Galicia, I., Sánchez, A. \& Robles, F.J. (2007). Relación de la depresión y rendimiento escolar en una población de adolescentes en el Estado de México. Horizontes, (14), 52-61.

Gómez, C. (2000). Identidades de género y feminización del éxito académico. Madrid: CIDE.

Hervás, G., Sánchez A. \& Vázquez, C. (2008). Intervenciones psicológicas para la promoción del bienestar. En: C. Vázquez \& G. Hervás (eds.). Psicología positiva aplicada (pp. 41-71). Bilbao: Desclée de Brouwer.

Labiano, M. (2007). Introducción a la psicología de la salud. En L. Oblitas. Psicología de la salud y calidad de vida. (2a ed., pp. 2-21). México: Thomson.

Lang, M. \& Tisher, M. (1997). Cuestionario de depresión para niños. Madrid: TEA.

Leyva; R., Hernández, A., Nava, G. \& López, V. (2007). Depresión en adolescentes y funcionamiento familiar. Revista Médica del Instituto Mexicano del Seguro Social, 45(3), 225-232.

Medina-Mora, M., Borge, G., Lara, C., Benjet, C., Blanco, J., Fleiz, C. \& Aguilar, S. (2003). Prevalencia de trastornos mentales y uso de servicios: Resultado de la Encuesta Nacional de Epidemiología Psiquiátrica en México. Salud Mental, 26(4), 1-16.

Organización Mundial de la Salud Ginebra (2004). Promoción de la salud mental: conceptos, evidencia emergente, práctica: informe compendiado. Francia: Fundación Victorian para la promoción de la Salud y la Universidad de Melbourne.

Organización Mundial de la Salud (2011). Depression. What is depression? Recuperado de: http://www.who. int/mental_health/management/depression/definition/en/\#

Pardo, G., Sandoval, A. \& Umbarita, D. (2004). Adolescencia y depresión. Revista Colombiana de Psicología, $13,13-28$. 
Pérez, M. \& Urquijo, S. (2001). Depresión en adolescentes. Relaciones con el desempeño académico. Psicología Escolar Educativa, 5(1), 49-58.

Peterson, C. \& Park, N. (2004). Classification and measurement of character strengths: Implications for Practice. En A. Linley y S. Joseph (eds.), Positive psychology in practice (pp. 433-446). New Jersey: Wiley.

Rush Universitary Medical Center (2011). Estadísticas relacionadas con los trastornos de salud mental. Recuperado de: http://www.rush.edu/spanish/sadult/ mentalhealth/facts.html

Ryff, C. (1995). Psychological well-being in adult life. Current Directions in Psychological Science, 4(4) 99. 104.

Salanova, M., Martínez, I., Bresó E., Llorens, S. \& Grau, R. (2005). Bienestar psicológico en estudiantes universitarios: facilitadores y obstaculizadores del desempeño académico. Anales de Psicología, 21(1), 170 180.

Seligman, M. (2008). Aprenda optimismo. México: Debolsillo.
Seligman, M., Steen, T., Park, N. \& Peterson, C. (2005) Positive psychology progress. Empirical validation of intervention. American Psychologist, 60(5), 410-421.

Sin, N. \& Lyubomirsky, S. (2009). Enhancing well-being and alleviating depressive symptoms with positive psychology interventions: A practice-friendly metaanalysis. Journal of Clinical Psychology: In Session, 65(5), 467-487.

Vázquez, C. (2009). La ciencia del bienestar psicológico. En: C. Vázquez y G. Hervás (eds.). La ciencia del bienestar. Fundamentos de una psicología positiva (pp. 13-46). Madrid: Alianza.

Vázquez, C. \& Hervás, G. (2008). Salud positiva: Del síntoma al bienestar. En: C. Vázquez y G. Hervás (eds.). Psicología Positiva Aplicada (pp. 17-39). Bilbao: Desclée de Brouwer.

Vázquez, C., Hervás, G., Rahona \& Gómez, D. (2009). Bienestar psicológico y salud: aportaciones desde la Psicología Positiva. Anuario de Psicología Clínica y Salud, (5), 15-28. 